\title{
Dissecting the mammalian synaptonemal complex using targeted mutations
}

\author{
Yael Costa $^{1} \&$ Howard J. Cooke ${ }^{2 *}$ \\ ${ }^{1}$ Division of Developmental Genetics and Stem Cell Research, MRC National Institute for Medical Research, \\ The Ridgeway, Mill Hill, London NW7 IAA, UK; ${ }^{2}$ Medical Research Council Human Genetics Unit, Western \\ General Hospital, Crewe Road, Edinburgh EH4 2XU, UK; Tel: +44-131-4678427; Fax: +44-131-4678456; \\ E-mail:howard.cooke@hgu.mrc.ac.uk \\ * Correspondence
}

Key words: meiosis, synaptonemal complex

\begin{abstract}
In many organisms completion of the first meiotic cell division depends on the correct assembly and disassembly of the synaptonemal complex (SC). This is a structure discovered a little over 50 years ago, which is formed by the close association of axes of homologous sister chromatid pairs. Its structure varies between organisms, although it retains a common tripartite organization in species as evolutionarily distant as budding yeast and humans. In mammals it is essential for crossover formation and completion of meiosis. Components of the mammalian SC have been identified only in the last 15 years, and mouse genetic approaches have started revealing the importance for this structure only in the past 5 years. Here we discuss the progress that has been made in the field of the mammalian SC and what approaches could be considered for its further study.
\end{abstract}

\section{Introduction}

Meiosis is the process of generating haploid gametes from diploid precursor cells. During this process mammalian cells perform the feat of aligning the homologous DNA molecules which form the chromosomes, generating recombination events between them and then segregating the four genomes in the replicated diploid cell into four nuclei. This is an astonishing achievement as it involves the manipulation of $4 \mathrm{~m}$ of DNA in a volume of about $400 \mu \mathrm{m}^{3}$, the equivalent to about 30 million kilometres in the volume of a table tennis ball. The mechanisms required to complete this process are complex. Distinct proteins assemble to form a structure, axial elements or $\mathrm{AE}$, with which each pair of sister chromatids is associated. When the AE connected to a pair of sister chromatids closely associates with the $\mathrm{AE}$ from the homologous sisters, they are termed lateral elements (LE). This association is what defines synapsis and is mediated by the transverse filaments (TF) and the central element (CE) as shown in Figure 1. Together, LE, TF and CE compose the SC. There is an intimate link between the assembly of the SC and the repair and recombination processes which follow the generation of double-strand breaks (DSB) (Keeney et al. 1997). DSB are required for homology searching and recombination which ultimately results (in mammals) in an average of between one and two genetic crossover events per chromosome pair.

Much of our current understanding of synapsis and recombination is derived from organisms such as Caenorhabditis elegans, Drosophila melanogaster and Saccharomyces cerevisiae with heavy emphasis on the latter. In these organisms cheap and rapid genetic screens have been used to identify genes involved in particular stages of meiosis, and biochemical 


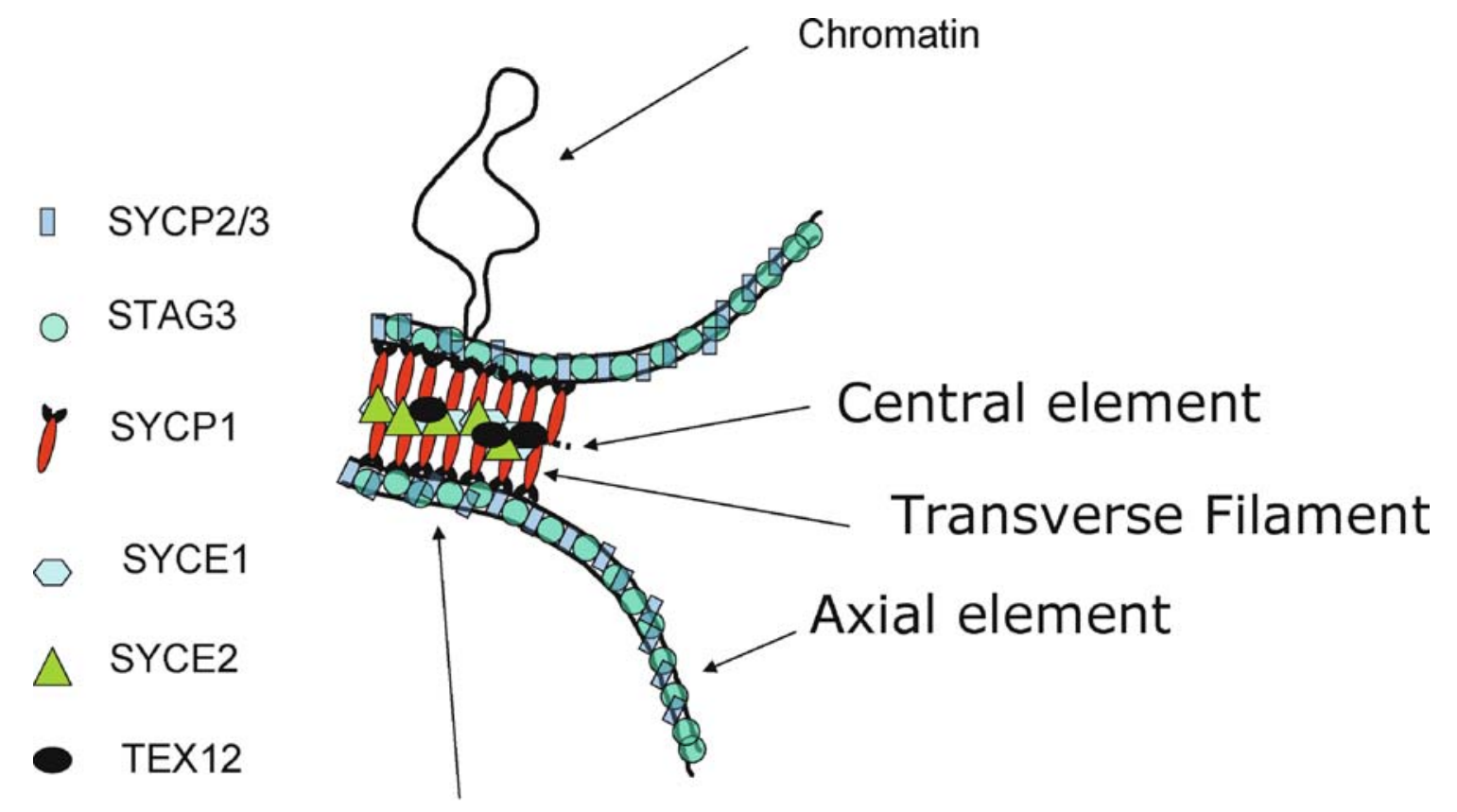

Lateral Element

Figure 1. Cartoon showing nomenclature and major components of the synaptonemal complex.

approaches have been used to define their function. One feature that has emerged is the conservation of structural motifs in SC proteins in the absence of easily detectable sequence conservation at genome or protein level. This contrasts with the repair proteins, which can be recognized at the sequence level in widely differing species. Mammalian systems have nonetheless some advantages despite the high cost and low speed of genetics and the lack of the meiotic culture systems which would permit siRNA and biochemically based approaches. Their genomes and hence chromosomes are larger than those of yeast and flies. This makes immunocytochemical analysis more powerful than in many other model organisms. Mouse genetics has been effectively used in combination with cytology to examine meiotic phenotypes produced as a result of targeted mutagenesis in embryonic stem cells. With the efforts to produce conditional knockouts of all mouse genes this approach is just beginning.

A primary driver of attempts to understand the processes of meiosis and particularly synapsis in mammals, is the fact that we are mammals. Infertility is estimated to affect $15 \%$ of couples, and a significant proportion of this is due to defects in meiosis. Although in some cases described at a cytological level, most of the cases of infertility due to meiotic problems escape diagnosis. Even when diagnosed and characterized clinically cases are unlikely to have the biochemical or genetic defect pinpointed. Aneuploidy is a further clinical manifestation of problems in meiosis, which is more common in females. In humans, when meiosis is arrested at the dictyate stage, chromosomes are held together by cohesion and chiasmata for decades. Failure of these processes to maintain correct chromosome segregation has catastrophic effects for the zygote.

In this review we will focus on mammalian synapsis and the use of targeted mutagenesis in mice to shed light on the molecular mechanisms involved in building the synaptonemal complex.

\section{Synaptonemal complex structure and assembly}

Meiosis can be viewed as a set of specific molecular events occurring after the completion of the last round of diploid DNA synthesis. Initiating with the formation of DSB, the normal process continues with 
the assembly of $\mathrm{AE}$ known to contain meiosisspecific proteins such as SYCP2, SYCP3 and cohesins, both meiosis-specific and canonical (Schalk et al. 1998, Prieto et al. 2001, Pelttari et al. 2001). The bulk of the DNA remains looped from these chromosome cores which gradually extend to encompass the whole length of the sister chromatids. DSB marked by extensive regions of phosphorylated histone $\mathrm{H} 2 \mathrm{AX}(\gamma \mathrm{H} 2 \mathrm{AX})$ have a $3^{\prime}$ extension which is thought to be involved in the homology search processes essential for homologue pairing, gene conversion and crossover (Rogakou et al. 1998, Hunter et al. 2001). These DSB are repaired and resolved with a small fraction of them resulting in a crossover and exchange of genetic information.

The role of cohesins in mammalian meiosis has been studied by using knockout mice for the meiotic cohesins SMC1 $\beta$ and REC8 (Revenkova et al. 2001, 2004, Eijpe et al. 2003). The role and regulation of cohesins in chromosome segregation during meiosis has been extensively reviewed, but in terms of synaptonemal complex formation the striking feature of both mutants is the overall reduction in the length of the axial elements (Klein et al. 1999, Nasmyth 2002, Revenkova \& Jessberger 2005). In the case of SMC1 $\beta$ knockout meiosis there is a high level of incomplete synapsis and the size of DNA looped out of the axial element is increased (Revenkova et al. 2004). This has been interpreted as a reduction in the amount of DNA packed into the $\mathrm{AE}$ but could equally be due to a difference in the degree of chromatin compaction in the loops caused by the absence of the meiotic cohesins. Despite these abnormalities, axial element proteins SYCP2 and SYCP3 are present, and where synapsis occurs the transverse filament protein SYCP1 is present. This phenotype is more extreme in the case of $\operatorname{Rec} 8$ mutants where absence of REC8 results in synapsis between sister chromatids (Xu et al. 2005). Together this suggests that these meiotic cohesins are not essential for axial element formation but are required for completion of synapsis between homologues. Temporal analysis of cohesin distribution suggests that REC8 is assembled into a SC-like structure before the other known components in wild-type meiosis, but this cannot be essential for SC formation (Eijpe et al. 2003). An important additional role for cohesins in stabilizing chiasmata and so chromosome attachment until anaphase of meiosis I in females is also likely, since analysis of Smc1 $\beta$ knockout mice shows reduction in recombination and increase in univalents which, as in human females, is agedependent (Hodges et al. 2005).

Major non-cohesin components of the SC have been demonstrated using antibodies raised against purified SC. Now named SYCP1-3, the second two proteins are components of the axial/lateral elements whereas SYCP1, present only when synapsis occurs, is a component of the transverse filaments that meet in the central element (Meuwissen et al. 1992, Lammers et al. 1994, Offenberg et al. 1998). SYCP2 and SYCP3 have been reported to be immunoprecipitated by antibodies recognizing cohesin subunits, suggesting a close but not necessarily direct interaction (Eijpe et al. 2000, Lee et al. 2003). Both proteins have been shown to interact in a two-hybrid system and more recently the interaction has been shown to be direct and to require the coiled coil domain of SYCP2 (Yang et al. 2006). SYCP2 is a $173 \mathrm{kDa}$ protein, expressed only in meiotic germ cells, with potential DNA binding motifs in addition to the coiled coil domain. Targeted mutagenesis has been used to generate mice producing SYCP2 with a deletion encompassing this region of the protein (Yang et al. 2006). In these animals the mutant protein is not associated with SYCP3. The males are sterile while the females have a reduced litter size. In the males, chromosomes do not synapse, although short stretches of SYCP1 staining can be detected by immunofluorescence and electron microscopy. The truncated protein is associated with axial elements, though these are abnormal and do not contain SYCP3. Synapsis, as reported by SYCP1 localization, is more complete in female meiosis, a common observation in mice with mutated meiotic genes. In these animals both sexes show SYCP3 present as aggregates in the nucleoplasm of meiotic cells, suggesting that its interaction with SYCP2 determines its incorporation into $\mathrm{AE}$; however, this conclusion is at odds with the phenotype of the Sycp3 knockout mouse in which SYCP2 is absent from the axes of chromosomes (see below). A possible explanation is that the mutation in the Sycp2 gene has resulted in a protein that is no longer dependent on SYCP3 for localization. The outcome of a null Sycp2 mutation would be informative.

SYCP3 itself is a small protein $(\sim 30 \mathrm{kDa})$ with potential coiled-coil and nucleotide binding motifs (Lammers et al. 1994). Male mice lacking this protein are sterile and exhibit germ cell death 
through apoptosis during zygotene (Yuan et al. 2000a). Spermatocytes do not form AE or SC which are recognizable by silver-based staining methods, but immunocytochemistry with antibodies to SYCP1 shows short, interrupted regions characteristic of partial synapsis. Staining for cohesin components reveals that some of these SYCP1 staining regions co-localize with AE-like cores, presumably where these are synapsed. Recombination proteins are still associated with these cores although levels in SYCP3-deficient animals may be reduced compared to wild-type. Again the phenotype of the null mutation is sexually dimorphic (Yuan et al. 2000b). Females are fertile but with reduced litter size, which is probably caused by elimination of embryos with aneuploidy (Yuan et al. 2002). Analysis of MI chromosomes showed an increased frequency of achiasmate chromosomes and a corresponding increase in the occurrence of aneuploid fertilized eggs. This effect was subtle as MLH1 foci, which mark sites of crossover resolution, were present with an overall small increase in number and variability between cells. A second abnormality of female synapsis in these Sycp3 null animals is the presence of breaks in the SYCP1 signal from synapsed chromosomes, suggesting that the structure of the LE is affected by the absence of this protein or by the associated absence of SYCP2.

The presence of SYCP1, a component of the transverse filaments, between the lateral elements is generally considered to mark synapsis (Figure 2). SYCP1 is a $111 \mathrm{kDa}$ protein that is predicted, based on its sequence, to have $\mathrm{C}$ and $\mathrm{N}$ terminal globular domains linked by an extended coiled coil region (Meuwissen et al. 1992, 1997). Also based on protein sequence, it has been suggested that the $\mathrm{C}$ terminus

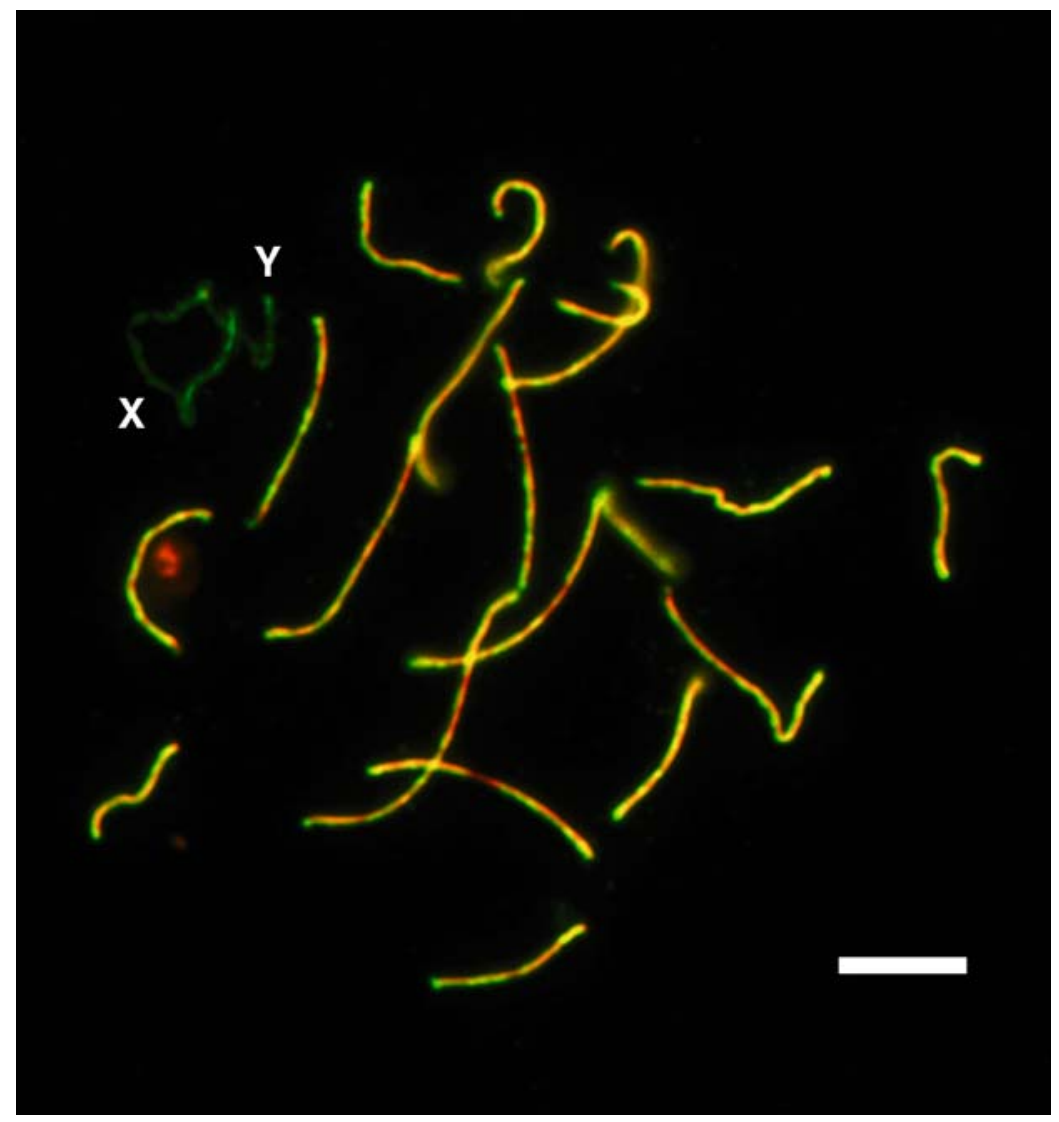

Figure 2. A normal male mouse pachytene spread with AE/LE represented by green immunolocalization of SYCP3 and SYCP1 immunolocalized in red. Note the lack of red signal on the unpaired $\mathrm{X}$ and $\mathrm{Y}$ chromosomes and the merged yellow signal present on fully synapsed autosomes. Scale bar represents $5 \mu \mathrm{m}$. 
could have DNA-binding properties. By using antibodies directed against $\mathrm{C}$ and $\mathrm{N}$ terminal regions in conjunction with immunogold labelling and electron microscopy, the $\mathrm{C}$ terminal domain has been located to the edge of the LE of the SC and the $\mathrm{N}$ terminal region to the CE (Schmekel et al. 1996). Yeast twohybrid assays have shown that the $\mathrm{N}$ termini of SYCP1 molecules can interact with each other and statistically based measurement of dimensions of SC and immunogold signals has led to the commonly illustrated model shown in Figure 1 (Liu et al. 1996). Transverse filaments composed of parallel dimers of SYCP1 associate through interdigitating $\mathrm{N}$ termini and this tetramer spans the gap between lateral elements.

A possible functional homologue of SYCP1 is present in yeast. This protein, ZIP1, locates to the transverse filaments (Sym et al. 1993). In most yeast cells during meiosis it also locates to a structure, the polycomplex, which may be a storage site for the protein (Sym \& Roeder 1995). Overexpression of ZIP1 increases the extent of polycomplex formation and electron microscopy shows a pattern of electron density reminiscent of stacked SC (Sym \& Roeder 1995). Similarly, overexpression of SYCP1 in cultured somatic cells produces aggregates (Ollinger et al. 2005). In the electron microscope these show periodic lines of differing electron densities with dimensions compatible to those of the SC. Changing the length of the coiled-coil region of the overexpressed SYCP1 results in changes in the width of these structures. Since these somatic cells do not express other meiotic proteins such as SYCP2 or 3 the implication is that $\mathrm{SC}$ dimensions are determined by the SYCP1 molecule, and that assembly could also be determined by this protein.

Not surprisingly, a knockout of this gene in male mice produces a failure of synapsis but autosomes are still paired and aligned, presumably through interactions such as strand invasions and doubleHolliday junctions which would normally result in crossover (de Vries et al. 2005). The exception to this is that the sex chromosomes are not associated. One possible explanation for this is that the limited region of homology to which the obligate crossover is confined does not contain enough of the interactions to stabilize any associations created in the absence of SC formation (Kipling et al. 1996). The other class of defects in male meiosis in these animals is in repair and recombination processes.
The phosphorylated form of histone $\mathrm{H} 2 \mathrm{AX}(\gamma \mathrm{H} 2 \mathrm{AX})$ is incorporated into chromatin for kilobases on either side of DSB and other DNA damage. In normal meiosis, leptotene cells show multiple sites of $\gamma \mathrm{H} 2 \mathrm{AX}$ incorporation but by pachytene these have disappeared and this histone modification is found only in the XY body. In Sycpl KO animals these are not all resolved and multiple $\gamma \mathrm{H} 2 \mathrm{AX}$ positive domains are still found. This is not unexpected based on in-vitro interactions shown between SYCP1 and RAD51 (Tarsounas et al. 1999). In addition, in these mutants, markers of recombination such as RAD51, RPA and MSH4 are present but are retained in comparison to wild-type cells. The markers of crossover, MLH1 and MLH3, are not found. This is consistent with recombination being blocked before crossovers can occur; in consequence, males are infertile. Females are also infertile, although the phenotype has not been fully reported (de Boer et al. 2006). However, based on the phenotype of the CE protein SYCE2 knockout (see below), it would be predicted to be similar to the male.

The proteins mentioned so far have been studied in meiosis because they are related to proteins with known function in mitosis or because they were detected biochemically in preparations enriched for synaptonemal complexes. More recently several laboratories have used approaches based on techniques such as library subtraction and microarrays to define genes expressed specifically in the testis (Wang et al. 2001, Maratou et al. 2004, Toure et al. 2005). In model organisms such as the mouse this can be coupled with measuring the onset of gene expression in the synchronous progression through the first wave of spermatogenesis and thus linking expression profiles to stages of germ cell development. Taking this analysis beyond the global scale, and looking at individual candidate genes, is laborious but has recently led to the discovery of three new components of the synaptonemal complex. These proteins, SYCE1, 2 and TEX12, are components of the CE which is formed when chromosome axes are synapsed (Costa et al. 2005, Hamer et al. 2006). To date they are the only known components confined to this structure together with the $\mathrm{N}$ terminal region of SYCP1. Immunocytochemistry has shown that in the absence of SYCP1 these proteins are delocalized from the chromosomes. Moreover, biochemical data show that the N terminus of SYCP1 interacts with both SYCE1 and 2, and that these proteins can interact with 
themselves and with each other, suggesting a possible structural role for the central element involving all these interactions (Costa et al. 2005). This has been confirmed by the finding that TEX12 interacts with SYCE2 and that it is likewise delocalized in Sycpl KO animals (Hamer et al. 2006).

The first phenotype of null mutations in a CE protein has been reported recently (Bolcun-Filas et al. 2007). In both male and female meiosis AE form with normal levels of cohesins and SYCP2 and 3. Homologous pairs of chromosomes are juxtaposed but synapsis does not occur. As is the case in the Sycpl knockout, the $\mathrm{X}$ and $\mathrm{Y}$ chromosome are not juxtaposed and sex body formation does not occur. $\gamma \mathrm{H} 2 \mathrm{AX}$ domains are not removed after zygotene and although RAD51, RPA and MSH4 are present, the markers of crossover MLH1 and MLH3 are absent. The phenotype in male is very similar to that in the Sycpl knockout. In either knockout both sexes are infertile. A significant difference between these two $\mathrm{SC}$ mutants is that, in the male Syce 2 knockout,
SYCP1 is absent from the AE except at occasional points of contact between pairs of $\mathrm{AE}$, which also contain SYCE1 (Figure 3). This suggests that, in contrast to the results from overexpressing SYCP1 in cell culture, the SC can not be formed in vivo by $\mathrm{N}$ and $\mathrm{C}$ terminal interactions of SYCP1 alone. Rather, these interactions set the spacing of the LE but are by themselves unstable, requiring SYCE1 for the increased stability reflected by the points of $\mathrm{AE}$ contact in the Syce2 knockout. Clearly, SYCP1 cannot bind to the $\mathrm{AE}$ without its incorporation into a CE, implying that interactions at the $\mathrm{N}$ terminus of this molecule affect interactions at its $\mathrm{C}$ terminus. A further inference is that these regions of SYCP1/ SYCE1 complexes can not be stably extended in the absence of SYCE2. This model for the assembly of the SC is illustrated in Figure 4.

In budding yeast, synapsis initiaties at discrete sites-the synaptic initiation complex (SIC) which contain a number of defined proteins termed ZIP2, 3 and 4 (Chua \& Roeder 1998, Agarwal \& Roeder

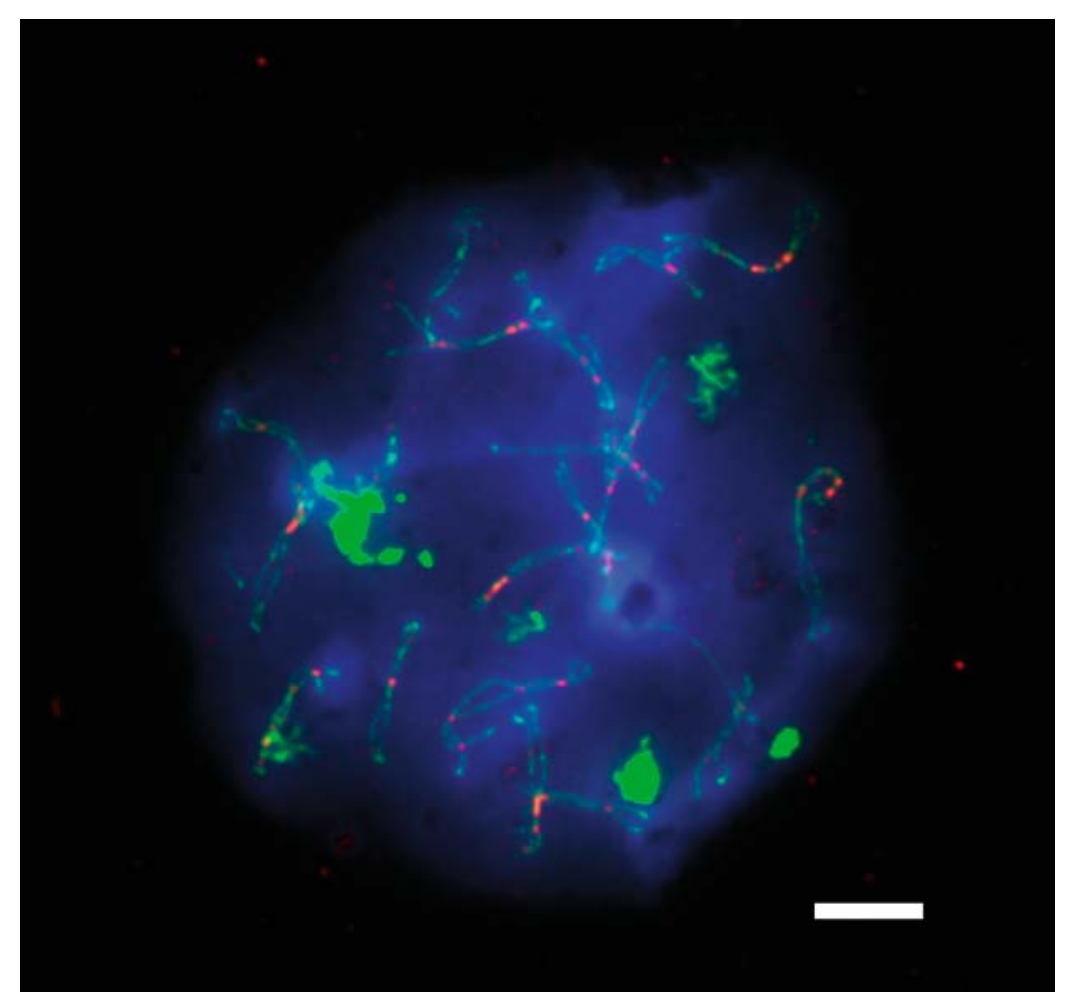

Figure 3. Disruption of synapsis in the absence of the CE protein SYCE2. Chromosomes are aligned but not synapsed except at occasional points of contact which contain SYCE1 and SYCP1. Axial elements are visualized by immunostaining for SYCP3 in green and SYCE1 is visualized in red. Scale bar represents $5 \mu \mathrm{m}$. 


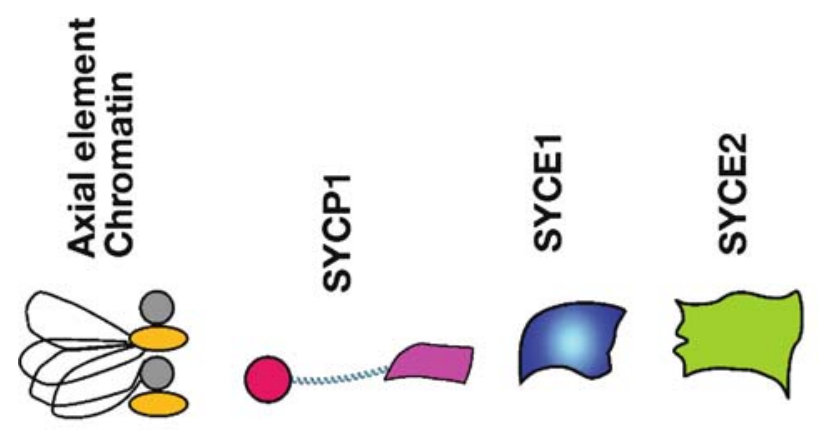

0

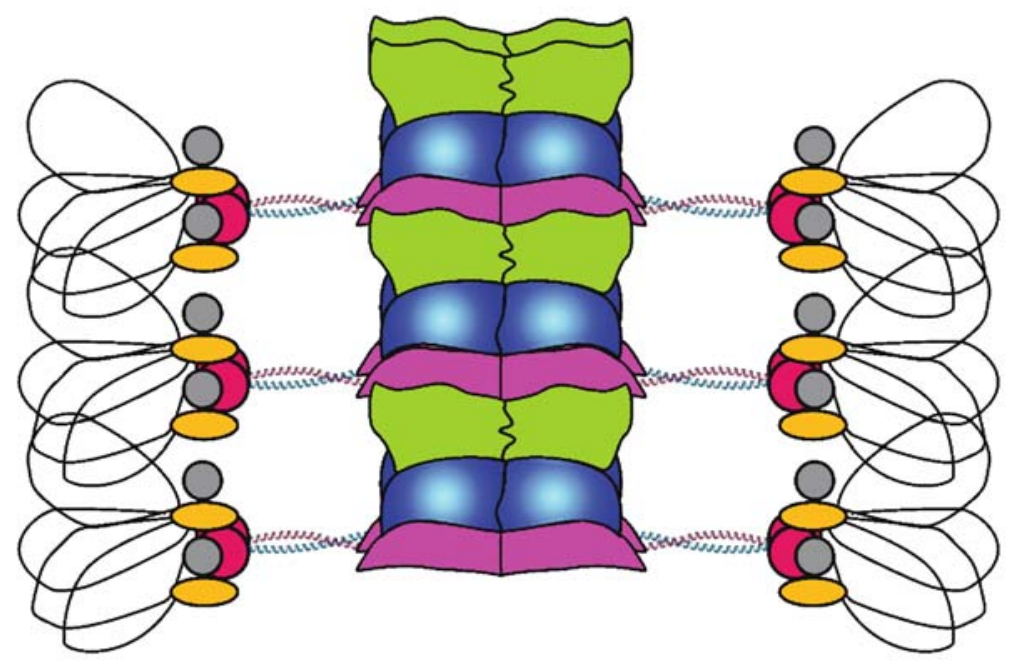

$\square$

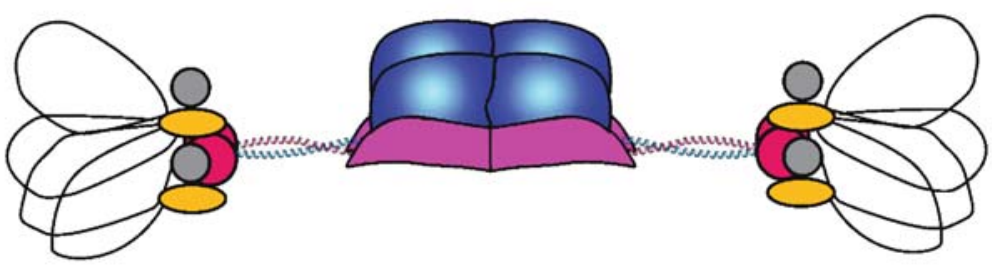

$\varangle$
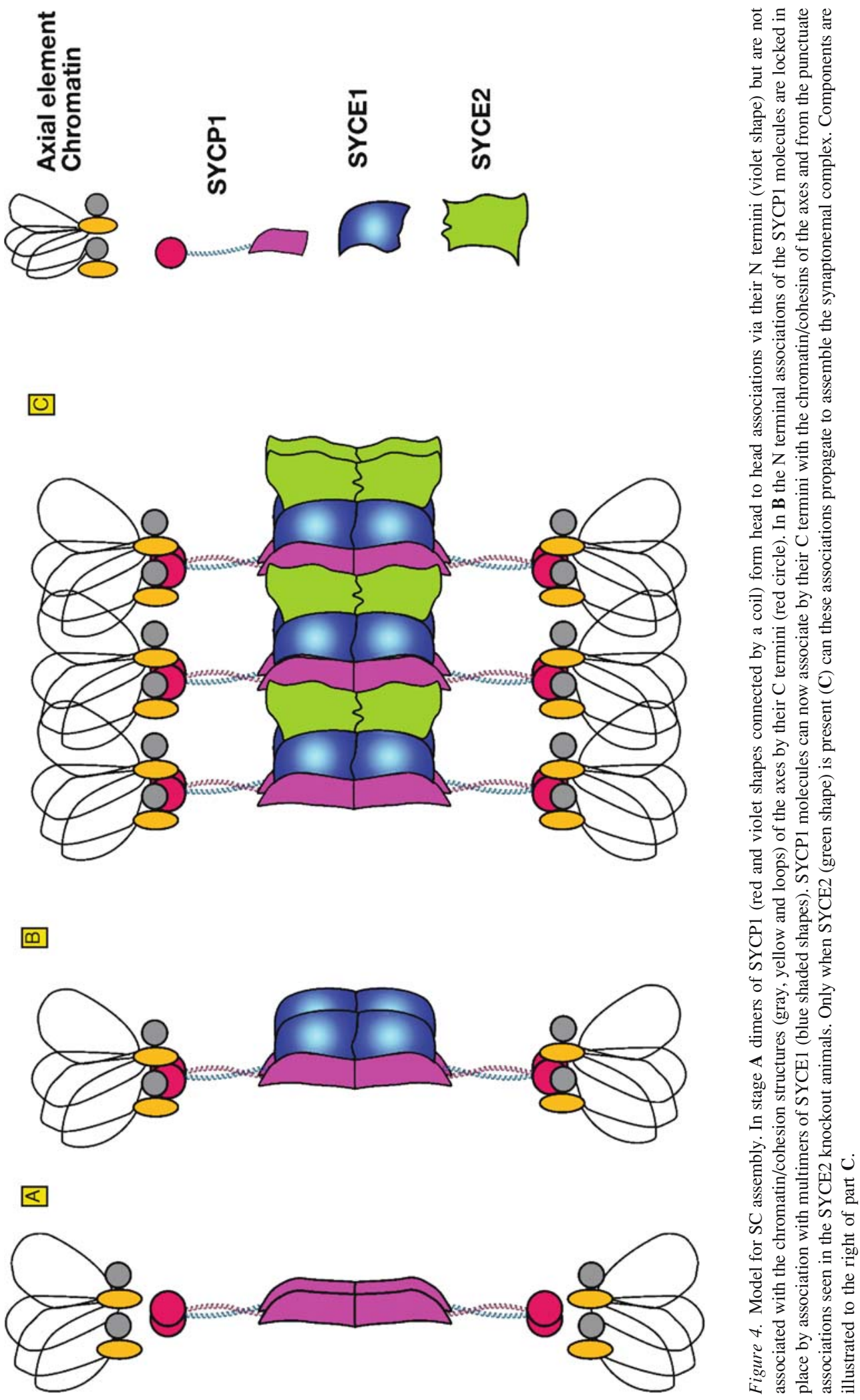
2000, Tsubouchi et al. 2006). The cytological distribution of SIC shows interference in the same way as does the genetic distribution of crossovers and, based on this and interaction/co-localization with Msh4 and 5, SIC have been inferred to mark sites of crossover. ZIP2 and 4 are dependent on ZIP3 for localization to axial elements, and the localization of Zip2 and 4 are mutually dependent, suggesting that they represent a functional unit (Tsubouchi et al. 2006). This is reminiscent of findings with SYCE1 and 2 and TEX12, but do the points of contact we see in the SYCE2 mutant animals represent mammalian SIC? Given the variability which we see in number and spacing from cell to cell this seems unlikely, but a definitive answer will require further experiments.

The picture of the SC with cohesins, both generic and meiosis-specific, interacting with the SYCP1-3 proteins and the three new constituents of the $\mathrm{CE}$, although complex, is incomplete. Other players have been found by chance-an example of this is the FKBP6 protein (Crackower et al. 2003). This protein is a member of a family of proteins which bind the immunosuppressive drug FK507. The human orthologue is involved in a contiguous genedeletion syndrome (Meng et al. 1998). When the mouse homologue was cloned and its expression pattern studied it was found to be a meiotic cellspecific protein which was located on synapsed, but also to a lesser extent on unsynapsed, chromosome regions. Disruption of the gene encoding this protein by targeted mutagenesis caused male mice to be infertile while females were unaffected. Among other abnormalities, synapsis was compromised with evidence for non-homologous pairing. Additionally there is biochemical evidence for interaction of SYCP1 and FKBP6 from mass spectrometry of immunoprecipitated protein and in Sycp3 knockout spermatocytes, the regions of synapsis detected by staining for SYCP1 were also stained by antibodies directed against FKBP6. However, the observation that FKBP6 is present on axial elements before synapsis suggests that this interaction is not essential for localization of FKBP6. In the rat a naturally occurring mutation causes azoospermia, and is the result of deletion of exon 8 of the Fkbp6 gene (Crackower et al. 2003). It is likely that both systematic and serendipitous work will produce more SC proteins, the role of which can be initially probed with the use of mouse models.

\section{Discussion}

Because many of the proteins described here function only during meiosis, and do not show haplo-insufficient effects, targeted mutagenesis in the mouse has been a useful method of generating novel meiotic phenotypes without resort to complex conditional strategies. These phenotypes give clues to the mechanisms involved, although this may not be unambiguous. As an example, REC8 expression patterns are consistent with it forming the basis of an AE scaffold on which other components such as SMC1 $\beta$, SYCP2 and SYCP3 assemble. However, the REC8 knockout animal forms AE, although they are clearly fragmented and undergo inter-sister chromatid rather than inter-homologue synapsis in both sexes. A further example of inconsistencies between mouse models is that of Sycp2/Sycp 3 mutants. Knockout of SYCP3 delocalizes SYCP2 from the remaining $\mathrm{AE}$ but introduction of a mutation into SYCP2 which blocks its interaction with SYCP3 results in delocalization of SYCP3 but not SYCP2. It will be necessary to have a deeper understanding of the mechanism before these differences can be reconciled.

Knocking out a single gene is a reductionist approach which may not be entirely appropriate to understanding the SC. As the name implies, this is a complex of proteins, and what the currently available range of knockouts demonstrate is that some features are remarkably robust-for example AE-like structures can be found in a variety of knockouts-but that complete function is not achieved without all components being present. In this situation dependencies cannot easily be resolved by knockouts. As an example, the relationships cited above for $\mathrm{CE}$ assembly do not fit into a simple, linear epistatic model, but instead are an example of co-dependence in building a complex of protein molecules all of which may be needed for function.

Although genetic approaches can provide information about the function of known components of a process such as synapsis in mouse, unlike in yeast and other simpler model organisms, they are difficult and expensive to use in the form of screens to define new players in the process. An alternative approach to this in mammalian systems has been to use the human population as a source of mutants. Human populations are relatively large and particularly well 
phenotyped, and this has been used to map and characterize genes and pathways. This has not so far been a productive approach in the case of infertility in general and meiosis in particular. The complexity of the infertility phenotype, and the difficulty of obtaining relevant tissue to generate a clear picture of pathology, is one reason for this; the large number of genes and small individual contribution of any one to the total of cases of infertility is another. Input in the opposite direction, from targeted mutagenesis to the human population, has been marginally more successful. Mutations have been reported in the Sycp3 gene of men with a meiotic arrest phenotype, although this has not been replicated in other populations and most recently mutations have not been found in the Fkbp6 gene (Stouffs et al. 2005, Zhang et al. 2005, Miyamato et al. 2006). The lack of a suitable cell culture system is a major hindrance to the study of meiotic processes in mammals. Genetic approaches in the mouse are developing rapidly, with consortia producing conditional null mutants for all known genes; but this still requires the production of mice, which is slow and expensive. A potential route around this could be the production of meiotic cells from pluripotent embryonic stem cells. Progress has been made recently with the production of haploid cells and mice from ES cells, but the overall efficiency is currently too low to exploit the system other than on a single-cell basis (Hubner et al. 2003, Toyooka et al. 2003, Geijsen et al. 2004, Nayernia et al. 2006, Guan et al. 2006). In addition it is not clear that these cells go through a normal meiosis (Novak et al. 2006). If efficiencies improve then a variety of approaches such as those based on siRNA could be used to rapidly screen for new components. Subtle mutations could also be introduced into known proteins to investigate mechanisms at a more detailed level than is currently feasible in the whole animal.

The next challenge is to move from having a list of components, locations and effects of null mutations to understanding the mechanistic basis for assembly and disassembly of the SC and its interactions with the proteins involved in repair and recombination. From comparisons of the relationship between synapsis and recombination in different organisms a general rule seems to be that organisms which make a large number of DSB require synaptonemal complex formation to successfully process those few DSB which will result in crossover. Mechanical forces have been suggested to be involved during this process, which a robust SC would need to withstand (Borner et al. 2004). However, it is also highly likely that the crossover complexes, visualized as late recombination nodules, contain many different proteins which are dependent on SC components for their functional assembly. Resolving such questions will require a combination of structure determinations coupled with the use of mutations to perturb function. Fortunately rapid progress in techniques such as cryo-electron microscopy and advanced optical microscopy are likely to be able to bridge the current gap between the protein sequence and immunocytochemistry of the mammalian SC.

\section{Acknowledgements}

Y.C. and H.J.C. are supported by the Medical Research Council of the UK.

\section{References}

Agarwal S, Roeder GS (2000) Zip3 provides a link between recombination enzymes and synaptonemal complex proteins. Cell 102: 245-255

Bolcun-Filas E, Costa Y, Speed R et al. (2007) SYCE2 is required for synaptonemal complex assembly, double strand break repair, and homologous recombination. J Cell Biol 176: 741-747.

Borner GV, Kleckner N, Hunter N (2004) Crossover/noncrossover differentiation, synaptonemal complex formation, and regulatory surveillance at the leptotene/zygotene transition of meiosis. Cell 117: 29-45.

Chua PR, Roeder GS (1998) Zip2, a meiosis-specific protein required for the initiation of chromosome synapsis. Cell 93: 349-359.

Costa Y, Speed R, Ollinger R et al. (2005) Two novel proteins recruited by synaptonemal complex protein 1 (SYCP1) are at the centre of meiosis. J Cell Sci 118: 2755-2762.

Crackower MA, Kolas NK, Noguchi J et al. (2003) Essential role of Fkbp6 in male fertility and homologous chromosome pairing in meiosis. Science 300: 1291-1295.

de Boer E, Stam P, Dietrich AJ, Pastink A, Heyting C (2006) Two levels of interference in mouse meiotic recombination. Proc Natl Acad Sci USA 103: 9607-9612.

de Vries FA, de Boer E, van den BM et al. (2005) Mouse Sycp1 functions in synaptonemal complex assembly, meiotic recombination, and XY body formation. Genes Dev 19: 1376-1389.

Eijpe M, Heyting C, Gross B, Jessberger R (2000) Association of mammalian SMC1 and SMC3 proteins with meiotic chromosomes and synaptonemal complexes [In process citation]. J Cell Sci 113: 673-682. 
Eijpe M, Offenberg H, Jessberger R, Revenkova E, Heyting C (2003) Meiotic cohesin REC8 marks the axial elements of rat synaptonemal complexes before cohesins SMC1beta and SMC3. $J$ Cell Biol 160: 657-670.

Geijsen N, Horoschak M, Kim K et al. (2004) Derivation of embryonic germ cells and male gametes from embryonic stem cells. Nature 427: 148-154.

Guan K, Nayernia K, Maier LS et al. (2006) Pluripotency of spermatogonial stem cells from adult mouse testis. Nature 440: 1199-1203.

Hamer G, Gell K, Kouznetsova A et al. (2006) Characterization of a novel meiosis-specific protein within the central element of the synaptonemal complex. J Cell Sci 119: 4025-4032.

Hodges CA, Revenkova E, Jessberger R, Hassold TJ, Hunt PA (2005) SMC1beta-deficient female mice provide evidence that cohesins are a missing link in age-related nondisjunction. Nat Genet 37: 1351-1355.

Hubner K, Fuhrmann G, Christenson LK et al. (2003) Derivation of oocytes from mouse embryonic stem cells. Science 300: 1251-1256.

Hunter N, Borner GV, Lichten M, Kleckner N (2001) GammaH2AX illuminates meiosis. Nat Genet 27: 236-238.

Keeney S, Giroux CN, Kleckner N (1997) Meiosis-specific DNA double-strand breaks are catalyzed by Spo11, a member of a widely conserved protein family. Cell 88: $375-384$.

Kipling D, Wilson HE, Thomson EJ et al. (1996) Structural variation of the pseudoautosomal region between and within inbred mouse strains. Proc Natl Acad Sci USA 93: 171-175.

Klein F, Mahr P, Galova M et al. (1999) A central role for cohesins in sister chromatid cohesion, formation of axial elements, and recombination during yeast meiosis. Cell 98: 91-103.

Lammers JH, Offenberg HH, van Aalderen M et al. (1994) The gene encoding a major component of the lateral elements of synaptonemal complexes of the rat is related to $\mathrm{X}$-linked lymphocyte-regulated genes. Mol Cell Biol 14: 1137-1146.

Lee J, Iwai T, Yokota T, Yamashita M (2003) Temporally and spatially selective loss of Rec8 protein from meiotic chromosomes during mammalian meiosis. J Cell Sci 116: 2781-2790.

Liu JG, Yuan L, Brundell E et al. (1996) Localization of the $\mathrm{N}$-terminus of SCP1 to the central element of the synaptonemal complex and evidence for direct interactions between the N-termini of SCP1 molecules organized head-to-head. Exp Cell Res 226: 11-19.

Maratou K, Forster T, Costa Y et al. (2004) Expression profiling of the developing testis in wild-type and Dazl knockout mice. Mol Reprod Dev 67: 26-54.

Meng X, Lu X, Morris CA, Keating MT (1998) A novel human gene FKBP6 is deleted in Williams syndrome. Genomics 52: 130-137.

Meuwissen RL, Meerts I, Hoovers JM, Leschot NJ, Heyting C (1997) Human synaptonemal complex protein 1 (SCP1): isolation and characterization of the cDNA and chromosomal localization of the gene. Genomics 39: 377-384.

Meuwissen RL, Offenberg HH, Dietrich AJ et al. (1992) A coiledcoil related protein specific for synapsed regions of meiotic prophase chromosomes. EMBO J 11: 5091-5100.

Miyamato T, Sato H, Yogev L et al. (2006) Is a genetic defect in Fkbp6 a common cause of azoospermia in humans? Cell Mol Biol Lett 11: 557-569.
Nasmyth K (2002) Segregating sister genomes: the molecular biology of chromosome separation. Science 297: 559-565.

Nayernia K, Nolte J, Michelmann HW et al. (2006) In vitrodifferentiated embryonic stem cells give rise to male gametes that can generate offspring mice. Dev Cell 11: 125-132.

Novak I, Lightfoot DA, Wang H et al. (2006) Mouse embryonic stem cells form follicle-like ovarian structures but do not progress through meiosis. Stem Cells 24: 1931-1936.

Offenberg HH, Schalk JC, Meuwissen RJ et al. (1998) SCP2: a major protein component of the axial elements of synaptonemal complexes of the rat. Nucl Acids Res 26: 2572-2579.

Ollinger R, Alsheimer M, Benavente R (2005) Mammalian protein SCP1 forms synaptonemal complex-like structures in the absence of meiotic chromosomes. Mol Biol Cell 16: 212-217.

Pelttari J, Hoja MR, Yuan L et al. (2001) A meiotic chromosomal core consisting of cohesin complex proteins recruits DNA recombination proteins and promotes synapsis in the absence of an axial element in mammalian meiotic cells. Mol Cell Biol 21: 5667-5677.

Prieto I, Suja JA, Pezzi N et al. (2001) Mammalian STAG3 is a cohesin specific to sister chromatid arms in meiosis I. Nat Cell Biol 3: 761-766.

Revenkova E, Eijpe M, Heyting C, Gross B, Jessberger R (2001) Novel meiosis-specific isoform of mammalian SMC1. Mol Cell Biol 21: 6984-6998.

Revenkova E, Eijpe M, Heyting C et al. (2004) Cohesin SMC1 beta is required for meiotic chromosome dynamics, sister chromatid cohesion and DNA recombination. Nat Cell Biol 6: 555-562.

Revenkova E, Jessberger R (2005) Keeping sister chromatids together: cohesins in meiosis. Reproduction 130: 783-790.

Rogakou EP, Pilch DR, Orr AH, Ivanova VS, Bonner WM (1998) DNA double-stranded breaks induce histone H2AX phosphorylation on serine 139. J Biol Chem 273: 5858-5868.

Schalk JA, Dietrich AJ, Vink AC et al. (1998) Localization of SCP2 and SCP3 protein molecules within synaptonemal complexes of the rat. Chromosoma 107: 540-548.

Schmekel K, Meuwissen RL, Dietrich AJ et al. (1996) Organization of SCP1 protein molecules within synaptonemal complexes of the rat. Exp Cell Res 226: 20-30.

Stouffs K, Lissens W, Tournaye H, Van Steirteghem A, Liebaers I (2005) SYCP3 mutations are uncommon in patients with azoospermia. Fertil Steril 84: 1019-1020.

Sym M, Engebrecht JA, Roeder GS (1993) ZIP1 is a synaptonemal complex protein required for meiotic chromosome synapsis. Cell 72: 365-378.

Sym M, Roeder GS (1995) Zip1-induced changes in synaptonemal complex structure and polycomplex assembly. J Cell Biol 128: 455-466.

Tarsounas M, Morita T, Pearlman RE, Moens PB (1999) RAD51 and DMC1 form mixed complexes associated with mouse meiotic chromosome cores and synaptonemal complexes. J Cell Biol 147: 207-220.

Toure A, Clemente EJ, Ellis P et al. (2005) Identification of novel $\mathrm{Y}$ chromosome encoded transcripts by testis transcriptome analysis of mice with deletions of the $\mathrm{Y}$ chromosome long arm. Genome Biol 6: R102.

Toyooka Y, Tsunekawa N, Akasu R, Noce T (2003) Embryonic stem cells can form germ cells in vitro. Proc Natl Acad Sci USA 100: $11457-11462$. 
Tsubouchi T, Zhao H, Roeder GS (2006) The meiosis-specific Zip4 protein regulates crossover distribution by promoting synaptonemal complex formation together with Zip2. Dev Cell 10: 809-819.

Wang PJ, Mccarrey JR, Yang F, Page DC (2001) An abundance of $\mathrm{X}$-linked genes expressed in spermatogonia. Nat Genet 27: 422-426.

Xu H, Beasley MD, Warren WD, van der Horst GT, McKay MJ (2005) Absence of mouse REC8 cohesin promotes synapsis of sister chromatids in meiosis. Dev Cell 8: 949-961.

Yang F, Fuente RL, Leu NA et al. (2006) Mouse SYCP2 is required for synaptonemal complex assembly and chromosomal synapsis during male meiosis. J Cell Biol 173: 497-507.
Yuan L, Liu JG, Hoja MR et al. (2002) Female germ cell aneuploidy and embryo death in mice lacking the meiosisspecific protein SCP3. Science 296: 1115-1118.

Yuan L, Liu JG, Zhao J et al. (2000a) The murine SCP3 gene is required for synaptonemal complex assembly, chromosome synapsis, and male fertility. Mol Cell 5: 73-83.

Yuan L, Liu JG, Zhao J et al. (2000b) The murine SCP3 gene is required for synaptonemal complex assembly, chromosome synapsis, and male fertility. Mol Cell 5: 73-83.

Zhang W, Zhang SZ, Xiao CY et al. (2005) [Possible association between 278C/A single nucleotide polymorphism of FKBP6 and idiopathic azoospermia]. Zhonghua Yi Xue Yi Chuan Xue Za Zhi 22: $10-13$. 